\section{Radicals initiated by gamma rays in selected amino acids and collagen}

\begin{abstract}
Calf skin collagen and three amino acids essential for its structure, namely glycine, L-proline and 4-hydroxyl-L-proline, were irradiated with gamma rays up to a dose of $10 \mathrm{kGy}$. Conversion of radicals over time or after thermal annealing to selected temperatures was monitored by X-band electron paramagnetic resonance (EPR) spectroscopy. Some experimental spectra were compared with signals simulated based on literature data from the electron nuclear double resonance (ENDOR) studies. The following phenomena were confirmed in the tested amino acids: abstraction of hydrogen atom (glycine, proline, hydroxyproline, collagen), deamination (glycine, hydroxyproline), decarboxylation (hydroxyproline). Chain scission at glycine residues, radiation-induced decomposition of side groups and oxidative degradation were observed in irradiated collagen. The decay of radicals in collagen saturated with water occurred at lower temperatures than in macromolecules having only structural water. The paramagnetic centres were the most stable in an oxygen-free atmosphere (vacuum). Radical processes deteriorated the structure of collagen; hence, radiation sterilization of skin grafts requires careful pros and cons analysis.
\end{abstract}

Keywords: amino acid $\bullet$ collagen $\bullet$ EPR spectroscopy $\bullet$ gamma rays

G. Przybytniak ${ }^{\bowtie}$, J. Sadło, M. Dąbrowska, Z. Zimek Institute of Nuclear Chemistry and Technology 16 Dorodna St., 03-195 Warsaw, Poland

E-mail: g.przybytniak@ichtj.waw.pl

Received: 12 November 2018

Accepted: 28 December 2018

\section{Introduction}

Tissue allograft is an indispensable material in reconstructive surgery which can be used to repair damaged parts of the body. Serious concerns related to the use of collagen-based allografts are the danger of transmitting various infections, which is why effective sterilization is an important factor in the clinical use of transplants. Gamma radiation is a recommended method for sterilization of biological tissues, ensuring microbiological safety [1-4] and the elimination of fungi, viruses, bacteria and spores. However, skin allografts when irradiated with a sterilization dose change the structure and biomechanical properties. The main component of skin allograft is cell-free collagen with porous architecture and favourable morphology $[5,6]$, but it is susceptible to radiation and oxidative degradation $[7,8]$.

Collagen is a structural, naturally occurring protein consisting of a triple helix. Each chain has a well-defined amino acid repeating sequence $(\mathrm{G}-\mathrm{X}-\mathrm{Y})_{\mathrm{n}}$, where $\mathrm{G}$ is glycine and $\mathrm{X}$ or $\mathrm{Y}$ is proline $(\mathrm{P})$ or hydroxyproline $(\mathrm{HyP})$. The abundance of these three amino acids in mammal skin is 329, 126 and 95 per 1000 residues, respectively [9].

The impact of ionizing radiation on collagen is still not fully understood. Chipara et al. and Bowes et al. reported that irradiation reduces molecular weight of protein due to backbone cleavage $[10,11]$. 
The process might be initiated by electron capture followed by dissociation of the $\mathrm{N}-\mathrm{C} \alpha$ bond [12]. On the other hand, another report suggests that the dominant collagen degradation proceeds via strand fission at the $\mathrm{C}(\mathrm{O})-\mathrm{CH}_{2}$ bond [13]. The modification of the chemical structure of peptides can also start from photochemically induced hydrogen transfer between hydrogen-bonded adjacent groups [14]. Nomura determined that solid state collagen contained in $1 \mathrm{~g}$ of protein $0-0.07 \mathrm{~g}$ of structural water, $0.07-0.25 \mathrm{~g}$ of bound water, $0.25-0.45 \mathrm{~g}$ of temporary bound water and free water [15]. High doses cause breaking of hydrogen bond network and changes in structural water, disrupting the molecular structure of collagen. In addition, decomposition along with increase in dose is accompanied by the formation of free amino acids [16]. During irradiation, collagen cross linking was also reported [17]. The effect depended on the irradiation conditions and the physical state of biological material.

Electron paramagnetic resonance (EPR) spectra of irradiated collagen are frequently unspecific, and their interpretation is still under discussion. To get closer to the full analysis of the radical processes in collagen, some amino acids that play an important role in maintaining their conformation were tested. Previous EPR studies revealed complex spectra attributed to several amino acid radicals. However, the well-known unusually stable alanine signal turned out to be a superposition of three components [18]. In addition, the analysis of EPR spectra of radicals in single crystals, which seems to be easier to interpret, brings many unresolved problems. They result from the coexistence of protonated/deprotonated forms of amino acids, the presence of many conformers of one molecule, significant discrepancies between calculated and experimental coupling constants, the presence of exchangeable and non-exchangeable protons, hydrogen-bonding networks, etc. For these reasons, quantum mechanical calculations performed by Ban et al. [19] often did not correspond to the electron nuclear double resonance (ENDOR) results that provided valuable information, especially when using isotope effect (hydrogen/deuterium).

At the INCT, we conducted EPR studies of calf skin collagen and amino acids being important residues of the protein, namely glycine, L-proline and 4-hydroxy-L-proline. The main purpose of the research was to identify collagen radicals based on EPR results. Biomolecules irradiated at ambient or liquid nitrogen temperature were studied in an air atmosphere. Irreversible changes initiated by gamma radiation in collagen were monitored by EPR spectroscopy in the range from $100 \mathrm{~K}$ to ambient temperature, whereas the amino acid spectra were observed during the long-term storage. The research focused on attempts to interpret complex EPR signals based on ENDOR results available in the literature, despite the fact that the information was in some cases incomplete due to broadening of some ENDOR lines and the lack of data on the interaction between nitrogen atoms and radical centres. The EPR spectra of amino acids and collagen were confronted to confirm/exclude some absorptions in the collagen signal and to facilitate, if possible, its interpretation.

\section{Materials and methods}

Amino acids, i.e. glycine, L-proline, 4-hydroxy-L-proline, and collagen extracted from calf skin and freeze-dried were purchased from Sigma Co. and used as received.

Microcrystalline powders of amino acids placed in vials were irradiated in a gamma cell (Gamma Chamber 5000, BRIT, Mumbai, India) with a dose of $10 \mathrm{kGy}$ at a dose rate of $4.3 \mathrm{kGy} /$ hour. To determine the exact dose absorbed, alanine dosimeter was used according to ISO/ASTM 51607:2013(E). The quartz tubes were filled with irradiated amino acids and closed. EPR measurements were performed immediately after exposure to gamma radiation and during the long-term storage in the dark at ambient temperature.

Collagen was irradiated at $77 \mathrm{~K}$ in closed EPR tubes. Three types of samples were tested: dry collagen (containing only structural water) in air, dry collagen in a vacuum and wet collagen saturated with water for 3 days in an atmosphere of $100 \%$ relative humidity.

The studies were performed using the X-band EMXplus Bruker EPR spectrometer. The apparatus was equipped with a cryostat (temperature control system EMX 41YT) and a high-sensitivity resonant cavity. G-factor was controlled using DPPH (1,1-diphenyl-2-picryl-hydrazyl) standard. The following parameters were used in EPR measurements: modulation amplitude $0.1 \mathrm{mT}$, sweep width $20.0 \mathrm{mT}$, resolution 2000 points and various microwave powers. The number of scans ranged from 1 to 8 depending on the intensity of the signal. Collagen spectra in the range of $100-370 \mathrm{~K}$ were registered using a controlled temperature annealing procedure. After heating, the samples were kept at the selected temperature for $5 \mathrm{~min}$ to achieve a thermal equilibrium.

The experimental spectra were analysed using the WINEPR Bruker software, while simulations were carried out applying the SimFonia Bruker software.

\section{Results and discussion}

Glycine (G)

Glycine irradiated at ambient temperature showed an intensive 1:2:1 triplet of isotropic hyperfine splitting (hfs) of $2.08 \mathrm{mT}$ originated from interaction of two equivalent protons and $g=2.0030$ (Fig. 1a). The spectrum was attributed to radical GII formed after deamination resulting from one electron reduction (Scheme 1). Changes in EPR signals were monitored for 17 months. After 1 month, the triplet decayed and a new, much more complex pattern appeared. Based on the differences in the peak-to-peak intensity of the centreline, it was determined that the contribution of triplet in the first spectrum was $83 \%$ (Fig. 1a, insert). Other radicals were stable 


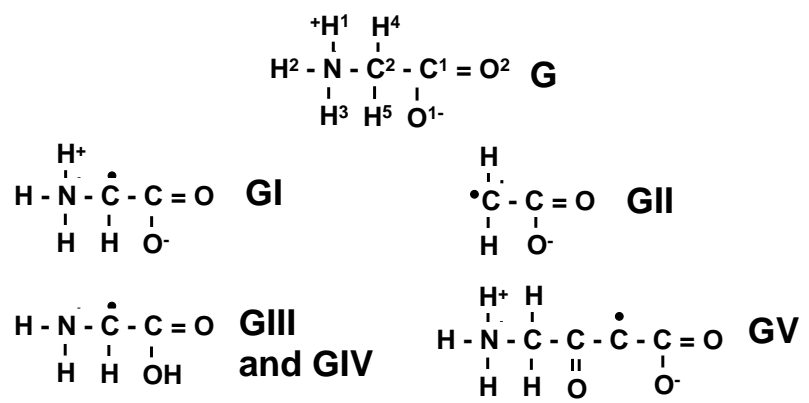

Scheme 1. Glycine and its radicals.

between the 1 st and 17 th month as indicated by the unchanged shape and amplitude of the signals. Due to confirmed stability of the signal, glycine was considered in the past as a potential EPR dosimeter of gamma radiation [20].

Despite many years of research, this well-resolved experimental spectrum has not been completely deconvoluted yet [21-24]. The main reason for difficulties in the interpretation of the results is the contribution of several components with different
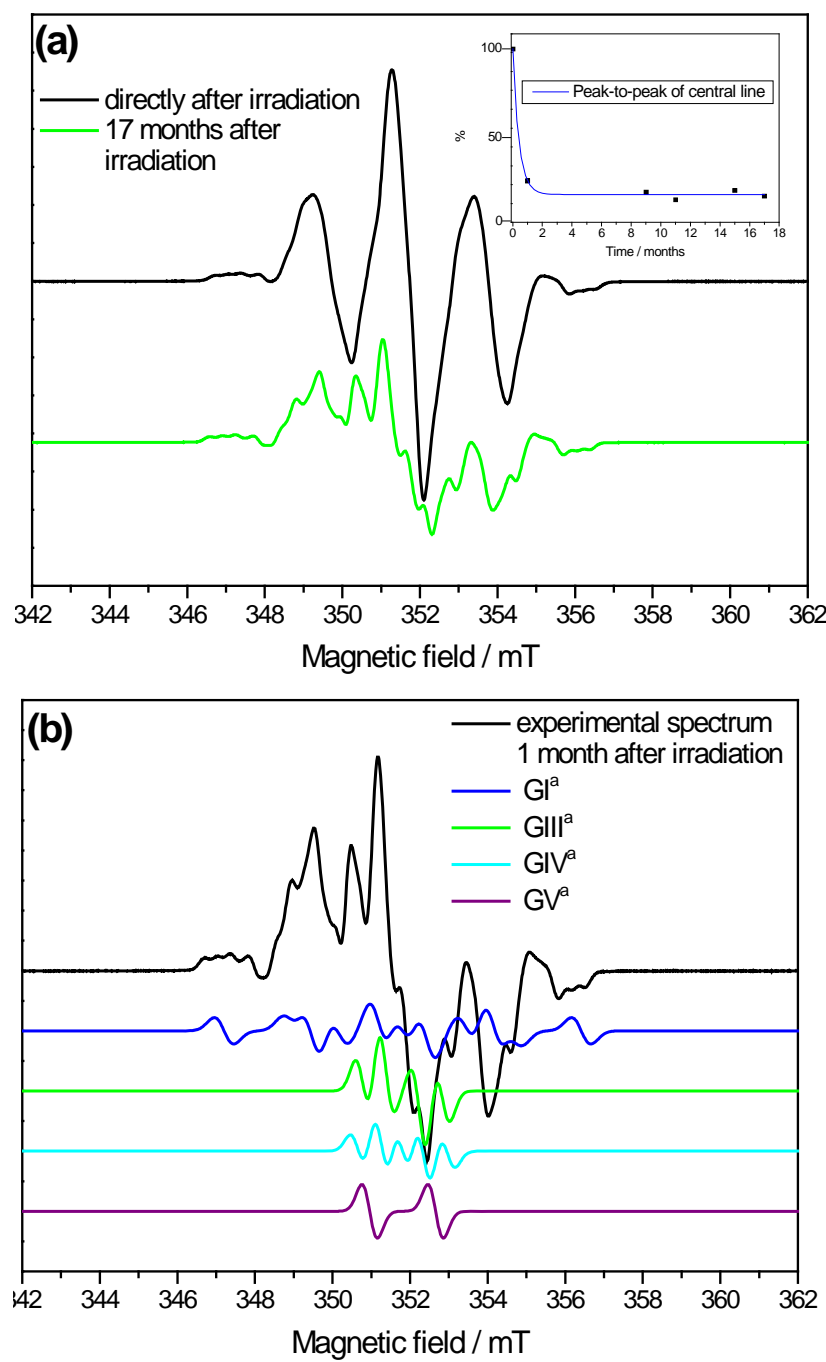

Fig. 1. (a) EPR spectra of glycine irradiated at ambient temperature (microwave power $0.6 \mathrm{~mW}$ ). Insert: Decrease in the amplitude of central line. (b) Comparison of glycine spectrum with spectra simulated based on ENDOR data [21]
Table 1. Hfs constants of glycine radicals expressed in $\mathrm{mT}$. If necessary, $\mathrm{MHz}$ was converted to $\mathrm{mT}$

\begin{tabular}{lccccc}
\hline $\begin{array}{c}\text { G radicals } \\
\text { (see Scheme 1) }\end{array}$ & $\mathrm{H}^{1}$ & $\mathrm{H}^{2}$ & $\mathrm{H}^{3}$ & $\mathrm{H}^{4}$ & $\mathrm{H}^{5}$ \\
\hline GI $^{\mathrm{a}}$ & 2.28 & 1.74 & 2.24 & 2.95 & \\
GII & & & & 2.08 & 2.08 \\
GIII \& GIV & 0.55 & 0.60 & & 0.87 & \\
GV $^{\mathrm{a}}$ & & & & 1.71 & \\
\hline
\end{tabular}

a-Ref. [21].

shapes but similar stability. Therefore, the experimental spectra were confronted with simulated signals based on the ENDOR data published by Sanderud and Sagstuen [21]. The EPR spectrum of a single crystal of glycine presented in their work is analogous to that shown in this paper. ENDOR studies carried out at ambient temperature revealed five radicals I-V as shown in Scheme 1. Each of the radical centres interacting with protons was characterized by a hyperfine coupling tensor. The influence of nitrogen atom was not evaluated experimentally, but for analysed radicals the coupling was negligible or small. Considering the isotropic hfs values reported in Ref. [21], the spectra of individual radicals were simulated and compared with the experimental EPR signal (Fig. 1b). The relevant parameters collected are summarized in Table 1.

The total width of the simulated GI spectrum resulting from non-equivalent interactions of one $\alpha$ proton and three exchangeable hydrogen atoms of amino group (proven by isotope effect H/D [21]) is comparable with the experimental EPR signal. The position of some EPR lines is also similar. The slight splittings $(<0.1 \mathrm{mT})$ of the external peaks might result from the interaction of unpaired spin and nitrogen atom which were undetected by ENDOR measurements.

The triplet of G II radical significantly decreased within a month. Spectra recorded later also revealed contribution of the signal. Stability of some GII molecules, which are sometimes called residual radicals, may result from their localization in defect-free crystals that maintain radical structure, preventing their further transformation.

GIII and GIV were suggested to be conformational isomers formed by oxidation [21, 24]. Their hfs constants are similar despite the different spatial structures. The intermediates are GI analogues formed by the proton transfer between amino and carboxyl groups. The presence of both paramagnetic species is likely, because the lines of the simulated signal are reflected in the experimental spectrum. The GV spectrum in the form of a doublet seems to be absent, because the analogous pattern was not found in the registered EPR signal. It was suggested that the intermediate was formed by the dimerization of deaminated products [21]. Considering the limited mobility of amino acid molecules in the microcrystalline powder, this process is unlikely, especially for small and medium doses.

In conclusion, the presence of G I-IV radicals in irradiated glycine was indirectly confirmed by comparison of the experimental EPR and ENDOR re- 

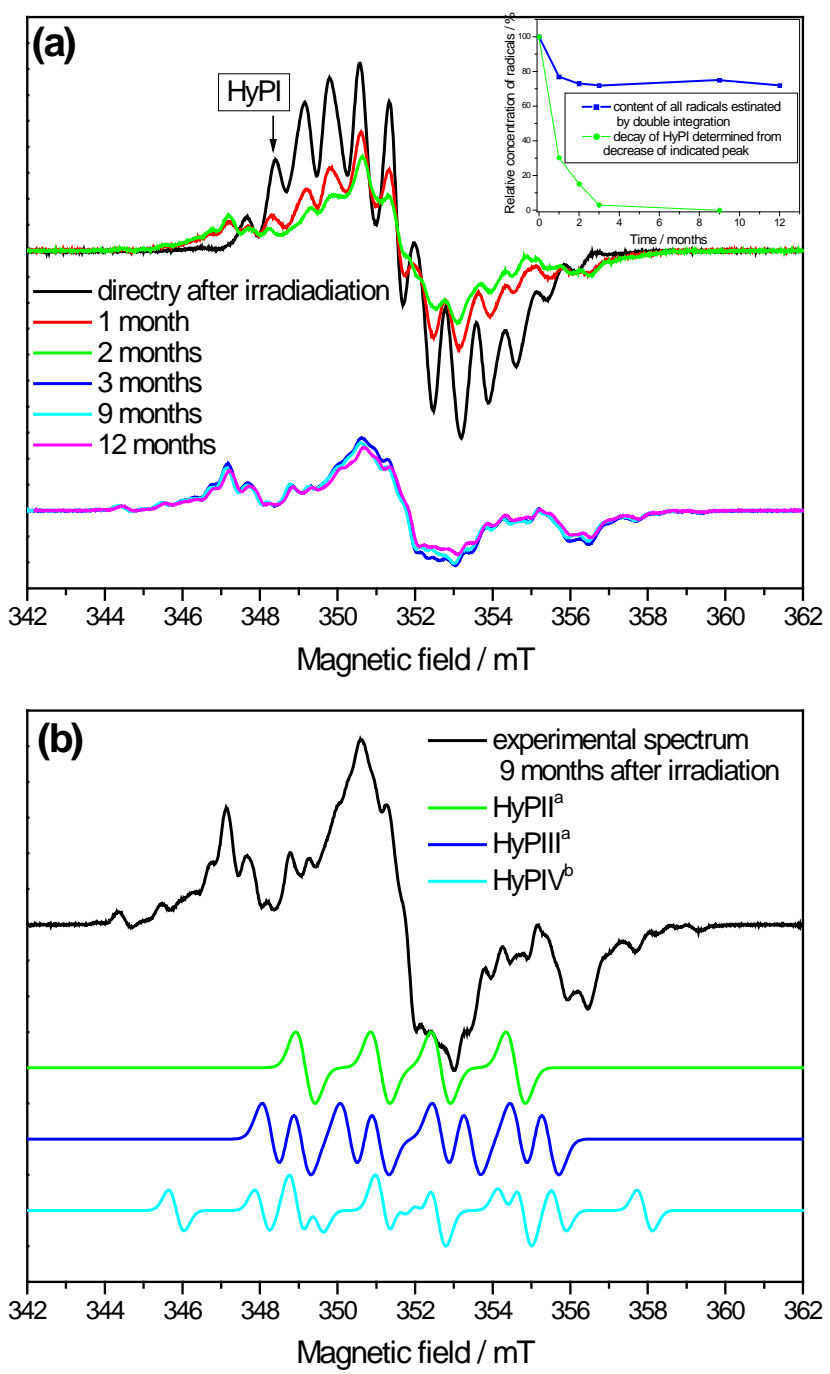

Fig. 2. (a) EPR spectra of HyP irradiated at ambient temperature and stored for 12 months (microwave power $0.6 \mathrm{~mW}$ ). Insert: Decay of radicals during storage. (b) Comparison of hydroxyproline spectrum with spectra simulated based on ENDOR data (a - Ref. [25], b - Ref. [26]).

sults. Interestingly, the stability of radicals, with the exception of GII, was similar, because no significant changes in the proportion between particular line groups were observed. An analogous phenomenon was confirmed for alanine radicals participating in the dosimetric EPR signal.

\section{Hydroxyproline (HyP)}

Hydroxyproline plays an important role in maintaining the triple helix in the collagen structure, making it more thermally stable [22]. The EPR spectrum of HyP irradiated at ambient temperature is rich and

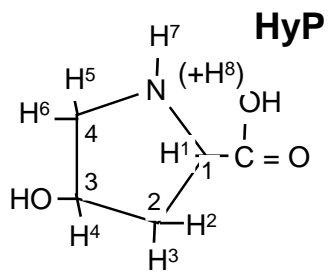<smiles></smiles>

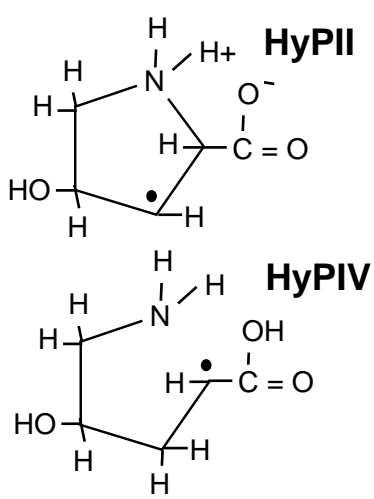<smiles>O=C([O-])C1CC(O)CN1[18OH]</smiles>

Scheme 2. Hydroxyproline and its radicals.

consists of 12 lines, separated by about $0.71 \mathrm{mT}$ (Fig. 2a). This radical is probably a product of decarboxylation (HyPI) in which unpaired spin interacts with five protons: two of them in the amino group are exchangeable and three are non-exchangeable. The signal can be simulated using the data presented in Table 2 [23]. Ban et al. predicted that radical cation HyPI formed as a result of decarboxylation might take the form of four conformers [19].

Theoretical studies based on density functional theory predicted a number of radicals derived from hydroxyproline [19]. With the exception of the radical anion, which was not analysed here due to high instability [14], other intermediates are depicted in Scheme 2.

With time, the signal changed significantly increasing the spectral range from $7.7 \mathrm{mT}$ to about $13.0 \mathrm{mT}$. The emerging absorption demonstrated many lines that were difficult to group into individual patterns. Hydroxyproline after dehydrogenation at C2 might form a HyP II radical showing hfs constants for one $\alpha$ and two $\beta$ protons. Theoretical studies suggested even eight conformers for HyP II in a form of non-zwitterions arising from various orientations of carboxylic and hydroxyl groups [19]. In ENDOR studies, Nelson [25] did not observe smaller hfs attributed to $\mathrm{H}^{1}$ proton. The corresponding spectrum simulated from these data seems to be present in a complex pattern detected upon long-term storage (Fig. 2b).

After abstraction of hydrogen atom from C3 carbon atom (HyPIII), the corresponding four

Table 2. Hfs constants of hydroxyproline radicals expressed in $\mathrm{mT}$. If necessary, MHz was converted to mT

\begin{tabular}{lccccccccc}
\hline HyP radicals & $\mathrm{H}^{1}$ & $\mathrm{H}^{2}$ & $\mathrm{H}^{3}$ & $\mathrm{H}^{4}$ & $\mathrm{H}^{5}$ & $\mathrm{H}^{6}$ & $\mathrm{H}^{7}$ & $\mathrm{H}^{8}$ & $\mathrm{~N}$ \\
\hline HyPI $^{\mathrm{a}}$ & 1.60 & 3.10 & 1.40 & & & & 0.70 & 0.70 & 0.35 \\
HyP II $^{\mathrm{b}}$ & & 1.93 & & 3.48 & & & & & \\
HyPIII $^{\mathrm{b}}$ & & 3.20 & 3.05 & & 2.22 & 3.61 & & & \\
HyPIV $^{\mathrm{c}}$ & 2.01 & 4.38 & 0.76 & & & & & & \\
\hline
\end{tabular}

a - Ref. [23], b - Ref. [25], c - Ref. [26]. 
conformers were predicted by the density functional theory [19]. A simulated spectrum constructed using ENDOR data [25] demonstrated that four $\beta$ proton couplings give a set of lines present in the EPR spectrum (Fig. 2b).

Radical HyP IV is a product of N-C bond scission which is considered as a process analogous to deamination. The simulation based on ENDOR parameters [26] is reflected in the experimental EPR spectrum. For this radical, there is no interaction of unpaired spin with nitrogen atom due to the opening of the pyrrolidine chain. Therefore, the use of ENDOR data to construct the EPR spectrum appears to be more reliable than for the previously discussed radicals.

Quantum mechanical calculations as well as ENDOR results did not completely account for experimental HyP signals [25, 26]. For example, in both cases, a distinctive triplet of hfs about $4.4 \mathrm{mT}$ was not discussed. This can be caused by the interaction of radiation-induced radical centres with different configurations and intermolecular hydrogen-bonding systems. Therefore, accurate deconvolution of the $\mathrm{HyP}$ spectrum requires analysis of the impact of crystalline environment.

\section{Proline (P)}

Proline is known as an amino acid useful in adaptive reactions to various types of harsh environmental conditions, e.g. oxidative stress [27]. The triplet arising after irradiation, for which the ratio of line intensity is $1: 2: 1$ and hfs is $2.06 \mathrm{mT}$, was assigned to the radical centre interacting with two $\beta$ protons of the methylene group (Fig. 3). The most probable intermediate PI shown in Scheme 3 was also proposed elsewhere [24]. The product is probably formed as a result of the conversion of radical anion of the parent molecule. The paramagnetic successor of PI radical has not been found yet; however, the most probable candidate is a product of decarboxylation since dissociation enthalpy of $\mathrm{C}-\mathrm{COOH}$ bond for PI radical is relatively low $(75 \mathrm{kcal} / \mathrm{mol})$ [28].

In addition to the dominant triplet, there are low-intensity outer lines separated by $10.3 \mathrm{mT}$. However, the central part of this spectrum is not resolved sufficiently to be interpreted.

\section{Collagen}

Collagen spectra recorded at cryogenic temperatures consisted of at least two components - a nonspe-<smiles>[1H][C@@H]1C(C(=O)[O-])CC[CH]N1[1H]</smiles>

Scheme 3. Proline radical.

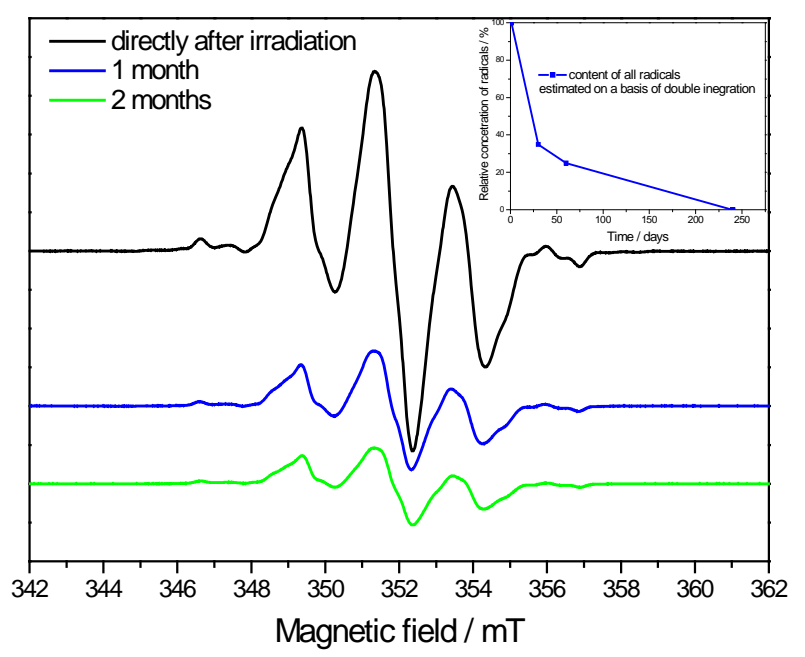

Fig. 3. EPR spectra of proline irradiated at ambient temperature and stored for 2 months (microwave power $0.6 \mathrm{~mW}$ ). Insert: Decay of radicals during storage.

cific broad singlet and a signal whose outer lines are visible on both sides of the central absorption (Fig. 4a). During thermal annealing, the wide singlet decreased, revealing other peaks. At low microwave power, a partly resolved quintet $(\mathrm{Q})$ was found show-
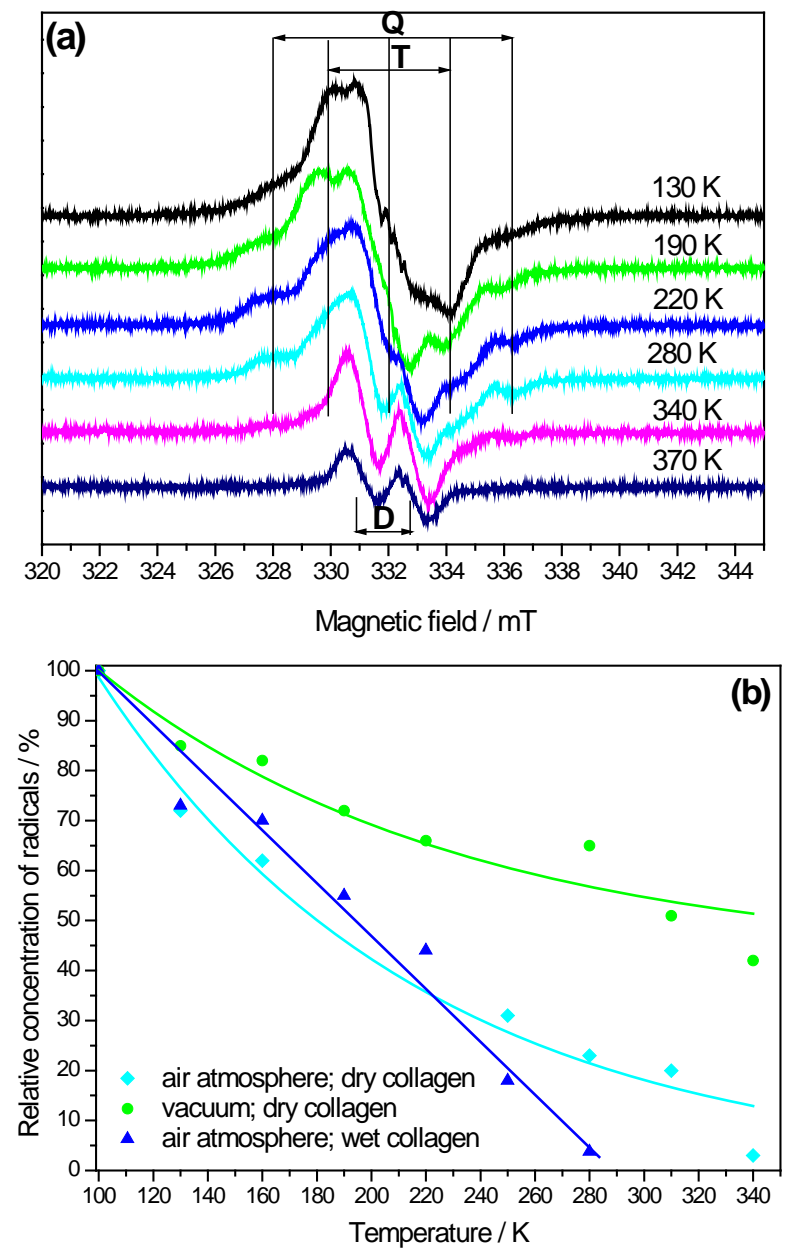

Fig. 4. (a) EPR spectra of dry collagen irradiated at $77 \mathrm{~K}$ in an air atmosphere and annealed to indicated temperatures (microwave power $1 \mathrm{~mW}$ ). (b) Decay of all radicals in irradiated collagens (dry, wet and in vacuum atmosphere) determined by double integration of experimental spectra. 
ing hfs of about $2.02 \mathrm{mT}$. This broad spectrum was assigned to the relatively stable radical, as the wings could be observed even at $340 \mathrm{~K}$. The presence of such a signal was also confirmed by Chipara et al. [10] and was attributed to radical at the end of the chain $\sim \mathrm{CH}_{2}-\mathrm{CH}_{2}{ }^{\circ}$ showing interaction with two $\alpha$ and two $\beta$ protons. The signal was stronger in the sequence of spectra recorded for wet collagen and in an oxygen-free atmosphere (results not shown). The intermediate exhibiting a quintet could be formed by radiolysis of certain side groups of collagen. At $280 \mathrm{~K}$, the doublet (D), showing hfs around $2.0 \mathrm{mT}$, related to glycine residue $\sim^{\circ} \mathrm{CH} \sim$, appeared as a secondary product. Since the intensity of the quintet remains unchanged up to $300 \mathrm{~K}, \sim \mathrm{CH}_{2}-\mathrm{CH}_{2}{ }^{\circ}$ could not be a precursor of $\sim^{\circ} \mathrm{CH} \sim$ radical. Therefore, in addition to these two alkyl radicals, another active centre at the end of the chain appeared to be present at low temperatures. Detailed analysis revealed that the second low- and high-field lines of quintet decreased with gradual thermal annealing in the range of $100-280 \mathrm{~K}$. These peaks probably originated not only from, discussed earlier, $\sim \mathrm{CH}_{2}-\mathrm{CH}_{2}$ radical but also from the triplet (T) whose middle line is covered by the central singlet. The spectrum (T) of irradiated collagen attributed to $\sim \mathrm{CH}_{2}$ showed hfs of about $1.98 \mathrm{mT}$ and indicated the cleavage of the collagen strain at the glycine residue. The other product of homolytic scission is $\sim(\mathrm{O}) \mathrm{C} \cdot$ radical that demonstrated a broad singlet. In fact, both radicals are able to abstract hydrogen atoms from the methylene group, which leads to the formation of $\sim^{\circ} \mathrm{CH} \sim$ showing the doublet. With increasing temperature, the total population of radicals decreased, which was determined by the double integration of experimental signals (Fig. 4b). Radicals in wet collagen decayed at lower temperatures compared with dry peptide. Anaerobic atmosphere (vacuum) additionally stabilized macroradicals, because under these conditions a doublet confirmed in the irradiated collagen was detected even after 5 months of storage in the dark. On the other hand, fully hydrated macromolecules create a three-dimensional hydrogen network actively participating in the transfer of protons, which facilitates the conversion/recombination of radicals.

No radicals found in collagen were similar to those detected in amino acids. There are two main reasons for these discrepancies. Dominant zwitterion structure of amino acids and intermolecular hydrogen bonds associated with this system determine the ionic nature of primary processes, which is not the case with collagen. In addition, peptide bond of collagen can play an important role in radical processes participating in the transfer of charge or hydrogen. Therefore, di- or tripeptides are more suitable models for testing than amino acids.

\section{Conclusions}

Several radicals were identified by EPR spectroscopy in glycine, L-proline and 4-hydroxy-L-proline irradiated with gamma rays at ambient temperature. In microcrystalline amino acid powders, the radicals are stable, but the interpretation of their spectra has been under debate for a long time due to the presence of many conformers, protonated to varying degrees and showing complex signals. The analysis of EPR results was based on their comparison with previously published ENDOR data.

Immobilization of atoms in nodes of crystalline lattices results in non-equivalence of interactions between unpaired spins and protons, while hfs constants characterized irradiated collagen do not reveal such discrepancies, because all $\alpha$ and $\beta$ hydrogen atoms show similar averaged values. This effect indicates significant local mobility even for dry macromolecules. The radicals found in collagen might initiate (i) damages in backbone substituents $\left(\sim \mathrm{CH}_{2} \mathrm{CH}_{2}{ }^{*}\right)$, which affect the structure of the peptide, (ii) strand breaks at glycine residues $\left(\sim \mathrm{CH}_{2}{ }^{\circ}\right)$ and (iii) release of hydrogen atom from glycine $\left(\sim \mathrm{CH}^{*} \sim\right)$. These phenomena have a detrimental effect on the collagen morphology, which should be taken into account before deciding on the methods of skin allograft sterilization.

EPR spectra of amino acids and collagen do not match each other. $\mathrm{C}(\mathrm{O})-\mathrm{N}$ units in collagen are different than in amino acids due to the formation of peptide groups that build the main chain eliminating the carboxylic groups and changing primary amines to secondary ones $(\mathrm{G})$ and secondary amines to tertiary ( $\mathrm{P}$ and $\mathrm{HyP}$ ). Therefore, surrounding of the carbon atoms situated between the peptide groups has a specific character. If radical centre is located at 2, 3 or 4 carbon atom of pyrrolidine ring, then analogous products would be present in both collagen and amino acids. However, the distinctive EPR spectra of HyP do not occur in collagen signals. It seems that more flexible conformations of peptide prefer other localizations of radical centres, including the main chain backbone.

Acknowledgment. The work was performed in the frame of the National Centre for Research and Development, Poland, STRATEGMED2/269807/NCBR/2015 (acronym: BIOOPA).

\section{References}

1. Samsell, B. J., \& Moore, M. A. (2012). Use of controlled low dose gamma irradiation to sterilize allograft tendons for ACL reconstruction: biomechanical and clinical perspective. Cell Tissue Bank., 13, 217-223. doi.org/10.1007/s10561-011-9251-7.

2. Balsly, C. R., Cotter, A. T., Williams, L. A., Gaskins, B. D., Moore, M. A., \& Wolfinbarger Jr., L. (2008). Effect of low dose and moderate dose gamma irradiation on the mechanical properties of bone and soft tissue allografts. Cell Tissue Bank., 9, 289-298. doi. org/10.1007/s10561-008-9069-0.

3. Greaves, L. L., Hecker, A. T., \& Brown, C. H. (2008). The effect of donor age and low-dose gamma irradiation on the initial biomechanical properties of human tibialis tendon allografts. Am. J. Sports Med., 36, 1358-1366. doi.org/10.1177/0363546508314394. 
4. Singh, R., Singh, D., \& Singh, A. (2016). Radiation sterilization of tissue allografts: A review. World J. Radiol., 8, 355-369. doi.org/10.4329/wjr.v8.i4.355.

5. Leroy, M., Labbé, J. F., Ouellet, M., Jean, J., Lefèvre, T., Laroche, G., Auger, M., \& Pouliot, R. (2014). A comparative study between human skin substitutes and normal human skin using Raman microspectroscopy. Acta Biomater., 10, 2703-2711. doi.org/10.1016/j. actbio.2014.02.007.

6. Pietrucha, K. (2015). Physicochemical properties of $3 \mathrm{D}$ collagen-CS scaffolds for potential use in neural tissue engineering. Int. J. Biol. Macromol., 80, 732-739. doi.org/10.1016/j.ijbiomac.2015.07.005.

7. Madison, S. A., McCallum, J. E. B., \& Rojas-Wahl, R. U. (2002). Hydroperoxide formation in model collagens and collagen type I. Int. J. Cosm. Sci., 24, 43-52. doi.org/10.1046/j.0412-5463.2001.00114.x.

8. Davies, M. J. (2016). Protein oxidation and peroxidation. Biochem. J., 473, 805-825. doi.org/10.1042/ BJ20151227N.

9. Szpak, P. (2011). Fish bone chemistry and ultrastructure: Implications for taphonomy and stable isotope analysis. J. Arch. Sci., 38(12), 3358-3372. doi: 10.1016/j.jas.2011.07.022.

10. Chipara, M., Reyes-Romero, J., Ignat, M., Constantinescu, B., \& Secu, C. (2003). ESR studies on collagen irradiated with protons. Polym. Degrad. Stab., 80, 45-49. doi.org/10.1016/S0141-3910(02)00381-6.

11. Bowes, J. H., \& Moss, J. A. (1962). The effect of gamma radiation on collagen1. Radiat. Res., 16, 211-223. doi.org/10.2307/3571153.

12. Syrstad, E. A., \& Tureček, F. J. (2005). Toward a general mechanism of electron capture dissociation. Am. Soc. Mass Spectr., 16, 208-224. doi.org/10.1016/j. jasms.2004.11.001.

13. Symons, M. C. R. (1996). Radicals generated by bone cutting and fracture. Free Rad. Biol. Med., 20(6), 831-835.

14. Smith, G. J. (1995). New trends in photobiology. Photodegradation of keratin and other structural proteins. J. Photochem. Photobiol. B-Biol., 27, 187-198. doi. org/10.1016/1011-1344(94)07104-V.

15. Nomura, S., Hiltner, A., Lando, J. B., \& Baer, E. (1977). Interaction of water with native collagen. Biopolym. J., 16, 231-246. doi.org/10.1002/ bip.1977.360160202.

16. Gauza-Włodarczyk, M., Kubisz, L., \& Włodarczyk, D. (2017). Amino acid composition in determination of collagen origin and assessment of physical factors effects. Int. J. Biol. Macromol., 104, 987-991. doi. org/10.1016/j.ijbiomac.2017.07.013.

17. Dziedzic-Goclawska, A., Kaminski, A., UhrynowskaTyszkiewicz, I., \& Stachowicz, W. (2005). Irradiation as a safety procedure in tissue banking. Cell Tissue Bank., 6, 201-219. doi.org/10.1007/s10561-0050338-x.

18. Ciesielska, B., Schultka, K., Penkowski, M., \& Sagstuen, E. (2004). EPR study of light illumination effects on radicals in gamma-irradiated L-alanine. Spectrochim. Acta Part A, 60, 1327-1333. doi: 10.1016/j.saa.2003.10.030.

19. Ban, F., Gauld, J. W., \& Boyd, R. J. (2000). Theoretical studies of the radiation products of hydroxyproline. J. Phys. Chem. A, 104, 8583-8592. doi.org/10.1021/ jp001692g.

20. Aboelezz, E., \& Hassan, G. M. (2018). Resolving the limitations of using glycine as EPR dosimeter in the intermediate level of gamma dose. Radiat. Phys. Chem., 145, 5-10. doi.org/10.1016/j.radphyschem.2017.12.006.

21. Sanderud, A., \& Sagstuen, E. J. (1998). EPR and ENDOR studies of single crystals of $\alpha$-glycine X-ray irradiated at 295 K. J. Phys. Chem. B, 102, 9353-9361. doi.org/10.1021/jp982932j.

22. Aydin, M., \& Osmanoglu, Y. E. (2011). EPR study of free radicals in amino acids derivatives by gamma rays. Rom. J. Phys. , 56, 1156-1161. doi.org/10.1016/j. msec.2017.06.012.

23. Kornacka, E. M., Przybytniak, G., \& Zimek, Z. (2018). Radicals initiated by gamma-rays in collagen and its main components. Radiat. Phys. Chem., 142, 4-8.

24. Brustolon, M., Chis, V., \& Maniero, A. L. (1997). New radical detected by HF-EPR, ENDOR, and pulsed EPR in a room temperature irradiated single crystal of glycine. J. Phys. Chem. A, 101, 4887-4892. doi. org/10.1021/jp970347x.

25. Nelson, W. H. (1988). ESR and ENDOR studies of radicals produced in hydroxyproline single crystals by $\mathrm{x}$-irradiation at low temperatures. J. Phys. Chem., 92, 554-561. doi.org/10.1021/j100313a060.

26. Nelson, W. H., \& Nave, C. R. (1981). ESR and ENDOR studies of radicals produced in hydroxyproline single crystals by $\mathrm{x}$ irradiation at low temperature. J. Chem. Phys., 74, 2710-2716. doi.org/10.1063/1.441440.

27. Matysik, J., Alia, Bhalu, B., \& Mohanty, P. (2002). Molecular mechanisms of quenching of reactive oxygen species by proline under stress in plants. Curr. Sci., 82, 525-532. www.jstor.org/stable/24105959.

28. Rawadieh, S., Altarawneh, I., Alateyat, H. B., \& Altarawneh, M. (2013). Theoretical study on the unimolecular decomposition of proline. Comput. Theor. Chem., 1018, 45-49. doi.org/10.1016/j. comptc.2013.05.034. 